\title{
Asthma as a nonlinear complex dynamic system: a novel approach to understand the temporal behaviour of chronic asthma and its response to $\beta$-agonists
}

\author{
ERS Romain Pauwels Research Fund 2007, sponsored by GlaxoSmithKline
}

\author{
Urs Frey \\ Division of Paediatric Respiratory Medicine, Dept of Paediatrics, Inselspital, Bern University Hospital and University of \\ Bern, Bern, Switzerland
}

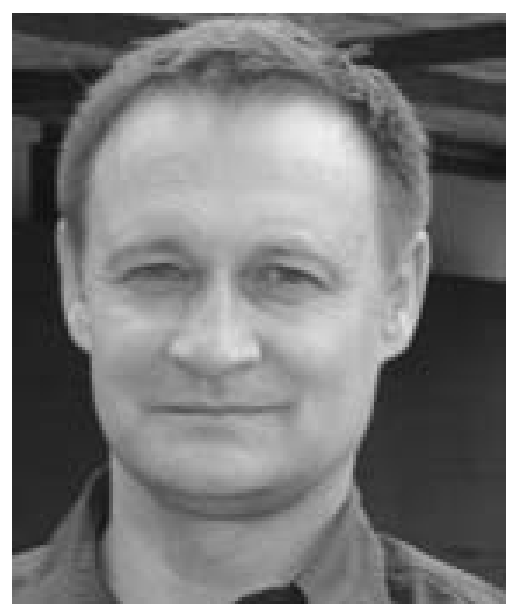

\section{SYNOPSIS OF MY JOB AND THE ROLE OF THE UNIT IN WHICH I WORK}

I am a paediatric pulmonologist, Professor and Head of the Division of Paediatric Respiratory Medicine at the University Children's Hospital in Bern, Switzerland. I graduated in 1989, wrote my MD thesis in paediatrics and trained as a paediatrician in Bern. I then spent some time in research and clinical training at Boston University (Boston, MA, USA), Hammersmith Hospital (London, UK) and Leicester University (Leicester, UK) and wrote a $\mathrm{PhD}$ in medical physics/biomedical engineering. My major research interests include the developmental physiology of newborns with wheezing disorders and asthma, and the potential role of environmental pollutants in lung growth and development. I was also involved in the development of new noninvasive lung function and nitric oxide measurement tests in infants. Such tests can be carried out in unsedated infants during natural sleep, and facilitate lung function studies in larger healthy cohorts and early recognition of infants at risk for later wheezing disorders and asthma. I was also significantly involved in the European Respiratory Society (ERS)/ American Thoracic Society standardisation of infant lung

STATEMENT OF INTEREST: None declared. function testing. My research has been supported by the Swiss National Science Foundation and by ERS long-term research fellowships. In 1999, I won the ERS Paediatric Respiratory Research in Europe Award and, in 2003, the Swiss Theodor-Kocher Research Award.

\section{ASTHMA AS A COMPLEX DYNAMIC SYSTEM}

The structure as well as the function of the respiratory system is complex. It includes subsystems involved in host defence, immunity and inflammation, as well as lung mechanics; these are inhomogeneous and irregular. More importantly, none of these subsystems function independently of each other but are highly interlinked, constituting a network with various tiers ranging from the molecular through cellular to organ level. Additional interactions occur within the organism, as well as within the external environment. Most studies that have led to advances in the understanding of the pathophysiology of the respiratory system have followed a "reductionist" or "analytical" approach, trying to isolate and identify specific mechanisms within this network that are responsible for certain processes or disease states. This analytical approach assumes linearity and divides the whole network into its components, in order to eventually understand the complete entity. A number of phenomena in biology, however, cannot be explained by such direct, deterministic relationships, since most biological systems are complex and function on the basis of interactions between a large number of components. The stimulation of a single component may affect a large number of other components in the network structure in a highly nonlinear manner. In such network constructs, it may even be that information from previous stimuli is stored (memory) and cumulative or delayed system effects may occur. Asthma as a complex chronic disease provides a good example. While the important mechanisms of asthma are known to include allergic inflammation, bronchial hyperreactivity and airway obstruction, this simple paradigm cannot explain why there is such a weak relationship between the strength of an adverse exposure or trigger and the clinical outcome (nonlinearity), for example in fatal asthma [1], or why bronchial hyperreactivity may persist for months after a single allergen exposure [2], or why people with occupational asthma may have persistent symptoms 
long after they have ceased to be exposed to the relevant environment [3]. The example of asthma suggests that the respiratory system, with its multitude of components and interactions, exhibits a stochastic behaviour coupled with complex nonlinear dynamics, leading to powerful fluctuations in physiological and clinical variables. Understanding the functioning of such complex network systems and predicting certain events, such as an asthma episode, is not possible within the realm of reductionist analysis. Rather, it requires a probabilistic systems approach, which is the principal topic of our work and which has been used in physics and biology for several decades.

Using mathematical methods derived from physics, we have recently proposed a new complex nonlinear systems approach accounting for memory effects to look at the pathophysiology and pharmacodynamics of chronic lung diseases, such as asthma [4-7]. We have found evidence for fractal statistical properties and long-range correlations in the fluctuations of long-range, twice-daily peak flow measurements in asthmatic adults [4]. These correlation properties of the signal can be characterised by the so-called detrended fluctuation analysis [8, 9], which measures the extent to which average variability in the past affects variability in the present. In such a temporal process with long-range correlations, the amplitudes of fluctuations that occurred in the past "influence" the current value. However, the influence of previous fluctuations decreases as the time interval over which the fluctuations are calculated increases, as described by a detrended fluctuation function, which forms the output of the analysis. Often, the function follows a power law which allows one to characterise the complex behaviour with a simple number, i.e. the slope of the function $(\alpha)$. A value of $\alpha=0.5$ represents a random, uncorrelated process. The higher $\alpha$ the more highly correlated the time series and the higher degree of internal "memory". In our previously reported study, we found that the mean $\pm S D$ (range) $\alpha$ for a daily peak expiratory flow (PEF) series of asthmatics was $0.78 \pm 0.19$ (0.40-1.32) and inversely correlated to asthma severity [4]; the closer the individual's $\alpha$ to 0.5 , the more unstable and unpredictable their asthma. Furthermore, regular treatment with the long-acting $\beta_{2}$-agent salmeterol strengthened these internal correlations, whereas short-acting $\beta_{2}$-mimetics administered regularly (q.i.d) drove the internal regulation of airway tone even more towards a random, less predictable process.

What are the consequences of these findings? The value of $\alpha$, and hence the strength of these long-range correlations, is likely to be influenced by the integrated contribution of intrinsic physiological airway control, as well as the effects of inflammatory, immunological and mechanical (remodelling) mechanisms. Although it is impossible to disentangle all the individual components of the system and its memory properties, it is possible to describe the overall dynamic behaviour of the disease in a comprehensive, integrated manner using this systems approach.

Internal long-range correlations are also important for the stability of the system, and are indicative of how the system is likely to behave in the future. In this asthma model, how prone the respiratory system is to future exacerbations is a key consideration. By knowing both past values of PEF embodied in the memory ( $\alpha$ of the PEF series) and the coefficient of variation of $\mathrm{PEF}$, it is possible to calculate the probability or risk (more precisely than conditional probability) that, given the current value of PEF, a severe episode of asthma (e.g. with a PEF of $<60 \%$ predicted) will occur within any time period (for example in the next month) [4].

\section{HOW WILL MY RESEARCH IMPACT ON CLINICAL PRACTICE?}

The impact of this research on clinical practice is reflected in the following ongoing work.

Application of the system approach to asthma phenotyping My previous work has focused on the prediction of exacerbations [4]. However, $\alpha$ could potentially be used to characterise different phenotypes of asthma. There is interesting evidence [10] that day-to-day peak flow variability is different in "coughvariant asthma" in comparison with "classical" asthma. Since long-range correlation $(\alpha)$ in PEF and variability (coefficient of variation) are not independent of each other, the latter work supports the hypothesis that $\alpha$ could be a new parameter for the characterisation of different asthma phenotypes. With the increasing interest in phenotype-specific treatment of asthma [11], a simple parameter $(\alpha)$ that helps to identify various asthma phenotypes may be of important future diagnostic value.

\section{Application of the systems approach to explaining response to $\beta$-agonists}

An increasing amount of work has shown that not all asthmatics react in the same way to $\beta$-agonists [12]. There has even been recent discussion concerning associations between mortality and long-term use of $\beta$-agonists [13]. This has been proposed to be related to genetic variation of the $\beta_{2^{-}}$ adrenoceptor (ADRB2), where it has been shown that asthmatic subjects who are homozygous for the arginine polymorphism at position 16 of their $\beta_{2}$-adrenoceptor have increased asthma symptoms associated with salbutamol treatment, and do not respond significantly to salmeterol treatment [14, 15]. The mechanisms by which this heterogeneity in response occurs are not well understood, and not easily related to previously published in vitro work. Also, no clear differences were seen in bronchial reactivity between the ADRB2 genotypes. These observations suggest that perhaps the conventional, linear perspective, i.e. of an adverse exposure (trigger) leading to inflammation and in turn bronchial hyperactivity, airway obstruction and finally symptoms, paints an inadequate picture of asthma. Using this approach for asthma, some evidence has been found that the heterogeneous response to $\beta$-agonist drugs in different genotypes is mediated through these intrinsic, airway control properties, as measured by $\alpha$. In addition, strong statistical associations have been found between $\alpha$ measured in a baseline state and clinical response to long-term $\beta$-agonist treatment. This suggests that $\alpha$ can be used to predict or identify individuals who may respond well or poorly to such treatment.

\section{IN SUMMARY}

If genetic, structural or functional risk factors can be identified that lead to highly unstable fluctuations in asthma dynamics, then we may be able to better understand asthma pathophysiology and identify patients at risk for future severe asthma attacks. This will also help us to better understand the complex interaction of genetic, environmental and intrinsic system dynamic factors involved in the pathogenesis of asthma and to 
better characterise and treat various asthma phenotypes. Furthermore, the findings in asthma may serve as a model for any other chronic disease with multiple influences. Finally, the systems approach of looking at chronic diseases potentially opens up new target areas for future drug studies.

\section{REFERENCES}

1 Plaza V, Serrano J, Picado C, Sanchis J. Frequency and clinical characteristics of rapid-onset fatal and near-fatal asthma. Eur Respir J 2002; 19: 846-852.

2 Cockcroft DW, Ruffin RE, Dolovich J, Hargreave FE. Allergen-induced increase in non-allergic bronchial reactivity. Clin Allergy 1977; 7: 503-513.

3 Lemiere C, Malo JL, Gautrin D. Nonsensitizing causes of occupational asthma. Med Clin North Am 1996; 80: 749-774.

4 Frey U, Brodbeck T, Majumdar A, et al. Risk of severe asthma episodes predicted from fluctuation analysis of airway function. Nature 2005; 438: 667-670.

5 Frey U, Maksym GN, Silverman M, Suki B. New approaches to the understanding of complex chronic lung diseases. In: Frey U, Gerritsen J, eds. Respiratory Diseases in Infants and Children. Eur Respir Mono 2006; 37: 345-360.

6 Frey U. Predicting asthma control and exacerbations: chronic asthma as a complex dynamic model. Curr Opin Allergy Clin Immunol 2007; 7: 223-230.

7 Suki B, Frey U. Temporal dynamics of recurrent airway symptoms and cellular random walk. J Appl Physiol 2003; 95: 2122-2127.
8 Peng CK, Havlin S, Stanley HE, Goldberger AL. Quantification of scaling exponents and crossover phenomena in nonstationary heartbeat time series. Chaos 1995; 5: 82-87.

9 Peng CK, Mietus J, Hausdorff JM, Havlin S, Stanley HE, Goldberger AL. Long-range anticorrelations and nonGaussian behavior of the heartbeat. Phys Rev Lett 1993; 70: 1343-1346.

10 Kang H, Koh YY, Yoo Y, Yu J, Kim DK, Kim CK. Maximal airway response to methacholine in cough-variant asthma: comparison with classic asthma and its relationship to peak expiratory flow variability. Chest 2005; 128: 3881-3887.

11 Bush A. Phenotype specific treatment of asthma in childhood. Paediatr Respir Rev 2004; 5: Suppl. A, S93-S101.

12 Taylor DR, Epton MJ, Kennedy MA, et al. Bronchodilator response in relation to $\beta_{2}$-adrenoceptor haplotype in patients with asthma. Am J Respir Crit Care Med 2005; 172: 700-703.

13 Martinez FD. Serious adverse events and death associated with treatment using long-acting $\beta$-agonists. Clin Rev Allergy Immunol 2006; 31: 269-278.

14 Taylor DR, Drazen JM, Herbison GP, Yandava CN, Hancox RJ, Town GI. Asthma exacerbations during long term beta agonist use: influence of beta 2 adrenoceptor polymorphism. Thorax 2000; 55: 762-767.

15 Taylor DR, Town GI, Herbison GP, et al. Asthma control during long-term treatment with regular inhaled salbutamol and salmeterol. Thorax 1998; 53: 744-752. 